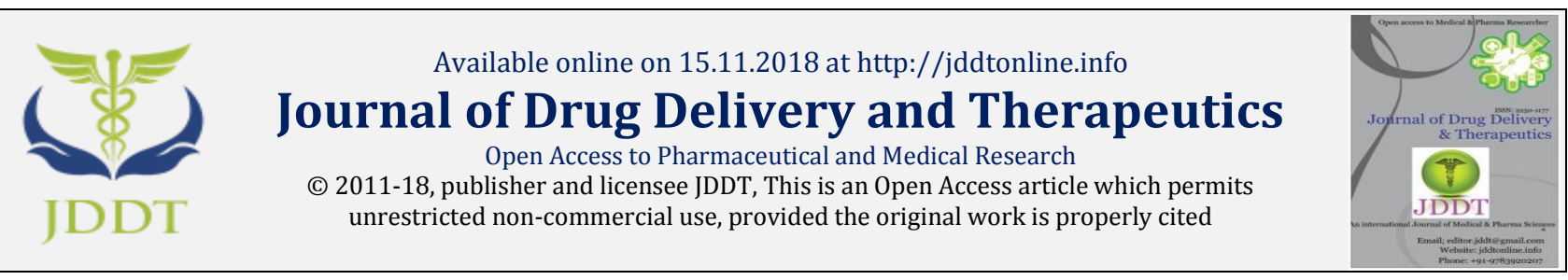

Open $\odot$ Access

Review Article

\title{
Majoon Suranjan: A Potent Unani formulation for Arthritis
}

\author{
Asim Ali Khan' ${ }^{1}$ Fouzia Bashir ${ }^{2 *}$, Jamal Akhtar ${ }^{3}$, Nighat Anjum ${ }^{3}$, Shah Alam², Sofia Naushin² \\ ${ }^{1}$ DG, Central Council for Research in Unani Medicine, New Delhi \\ 2 Research Associate, Central Council for Research in Unani Medicine, New Delhi \\ ${ }^{3}$ Research Officer, Central Council for Research in Unani Medicine, New Delhi
}

\begin{abstract}
Unani System of medicine is one among the oldest systems that prevails till date with its efficient drugs derived from animal, plant and mineral resources. Over 2400 years ago the father of medicine, Hippocrates practiced it, however His medicine included a great deal of ancient Egyptian medicine as well as important components of the ancient Mesopotamian traditions. In Unani system of medicine, arthritis is described under a broad term Waja-ul-Mafasil which encompasses entire joint disorders like inflammatory, non-inflammatory, infectious, metabolic and other musculoskeletal disorders. A large number of drugs, single and compound formulations, have been mentioned in the context of the treatment of Waja-ul-Mafasil. Among them, Majoon Suranjan is one of the most reputed poly pharmaceutical preparations of Unani system of Medicine used in Waja-ul-Mafasil. It is attributed to Indrumakhas of Greece (Andromachos, the elder, court physician to King Nero) who formulated it in consultation with several o ther philosophers of his time. This Unani compound formulation contains twenty one ingredients, which is used as digestive, purgative, anti inflammatory, stomachic, deobstruent, antiarthritic, nervine tonic. It is also indicated for use in phlegmatic diseases.
\end{abstract}

Keywords: Majoon Suranjan, Ingredients, Arthritis, Unani Medicine.

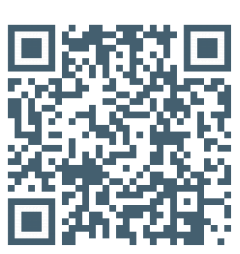

Article Info: Received 01 Oct, 2018; Review Completed 05 Nov 2018; Accepted 11 Nov 2018; Available online 15 Nov 2018

\section{Cite this article as:}

Khan AA, Bashir F, Akhtar J, Anjum N, Alam S, Naushin S, Majoon Suranjan: A Potent Unani formulation for Arthritis, Journal of Drug Delivery and Therapeutics. 2018; 8(6):351-355 DOI: http://dx.doi.org/10.22270/jddt.v8i6.2149

*Address for Correspondence:

Fouzia Bashir, Research Associate, Central Council for Research in Unani Medicine, New Delhi

\section{INTRODUCTION}

Unani System of medicine is one among the oldest systems that prevails till date with its efficient drugs derived from animal, plant and mineral resources. Over 2400 years ago the father of medicine, Hippocrates practiced it, however His medicine included a great deal of ancient Egyptian medicine as well as important components of the ancient Mesopotamian traditions. The methods of treatment according to Unani System of Medicine is divided into four different parts namely dietotherapy (Ilaj-Bil-Ghiza), regimenal therapy (Ilaj-Bil Tadbeer), surgery (Ilaj-Bil-Yad) and pharmacotherapy (Ilaj-Bil-Dawa) 8, 9, 12, 21, 23, 24. Considering pharmacotherapy, both single and compound drugs are being used. Among the polyherbal drugs, Majoon Suranjan (MS) is a formulation that is used in the Unani system of medicine and is being prescribed from centuries with great reputation for treatment of Arthritis and other joint disorders. According to different Unani Qarabadeen's (Pharmacopoeias) this compound formulation is used as digestive, purgative, anti inflammatory, appetizer, stomachic, deobstruent, antiarthritic, nervine tonic 7, 29, 32 . It is also indicated for use in phlegmatic diseases $13,19,21,27$.

\section{Prescription}

According to some of the Classical Unani Books, the original preparation of Majoon Suranjan contained only seven ingredients viz, Asaroon (Valeriana wallichii), Zanjabeel (Zingiber officinalis), Zeera Siyah (Carum Carvi), Darfilfil (Piper longum), Sana Maki (Cassia angustifolia), Suranjan (Colchicum luteum) and Honey 4, 5, 9, 10, 29, 36. However, there are certain variations regarding the weight and number of ingredients of the compound in different Unani formulations. The formula for preparation of Majoon Suranjan in National Formulary of Unani Medicine (NFUM) contains twenty one ingredients with sugar or honey as a base, whereas in Hamdard Pharmacopoeia of Eastern Medicine, the number of ingredients is same as that of NFUM but differs in dosage 22 . 
Table 1: Formula of Preparation of Majoon Suranjan according to NFUM 2, 28, 34, 43

\begin{tabular}{|l|l|}
\hline Barg e Hina khushk (Lawsonia inermis) & $15 \mathrm{gms}$ \\
\hline Badiyan (Foeniculum vulgare) & $15 \mathrm{gms}$ \\
\hline Asarun (Valeriana wallichii) & $20 \mathrm{gms}$ \\
\hline Beekh e Kibr (Caparis spinosa) & $20 \mathrm{gms}$ \\
\hline Post halela Zard (Terminalia Chebula) & $70 \mathrm{gms}$ \\
\hline Turbud Safaid (Ipomoea Turpethum) & $160 \mathrm{gms}$ \\
\hline Tukhm Karafs (Apium graveolens) & $15 \mathrm{gms}$ \\
\hline Chita Lakdi (Plumbago zeylanicum) & $20 \mathrm{gms}$ \\
\hline Zanjabeel (Zingiber officinalis) & $30 \mathrm{gms}$ \\
\hline Saqmunia (Convolvulus scammonia) & $30 \mathrm{gms}$ \\
\hline Samundar Jhag (Cuttlefish bone) & $15 \mathrm{gms}$ \\
\hline Suranjan Shireen (Colchicum luteum) & $60 \mathrm{gms}$ \\
\hline Sana (Cassia angustifolia) & $120 \mathrm{gms}$ \\
\hline Satar Farsi (Origanum vulgare) & $15 \mathrm{gms}$ \\
\hline Filfil Safaid (Piper album) & $15 \mathrm{gms}$ \\
\hline Kishneez Khushk (Coriandrum sativum) & $30 \mathrm{gms}$ \\
\hline Gul e Surkh (Rosa damascena) & $30 \mathrm{gms}$ \\
\hline Mahi Zehraj (Verbascum thapsus) & $20 \mathrm{gms}$ \\
\hline Namak Sambhar (Lake salt) & $15 \mathrm{gms}$ \\
\hline Roghan Arandi (Ricinus communis) & $120 \mathrm{gms}$ \\
\hline Qiwam Shakar Safaid (White sugar syrup) & $2.150 \mathrm{~kg}$ \\
& \\
\hline
\end{tabular}

\section{Method of Preparation}

For making Majoon or any of its allied preparations, Qiwam (base) of different consistencies (tar) is generally made. It depends on the nature of ingredient drugs to be used. The qiwam is generally made by adding Aab (water), Araq (distillate) or Aab e samar (fruit juices), etc in any of the bases of purified honey, sugar, candy or jaggery etc and boiled over a low heat till it acquires a required consistency. The bases are generally purified by adding Aab e lemu (lemon juice), Satt e lemu (lemon extract), or Shibb e yamani (Alum) before making qiwam. Afterwards the ingredients are mixed in qiwam to prepare Majoon. Qiwam for majoon is of two tar (consistency) 11, 26, 27, 30. Majoon Suranjan is made on the same principle while using water with sugar or honey as a base and boiled over low fire till qiwam (consistency) of two tar is achieved $32,33$.

All the dry ingredients, after being ground together and sieved through 80-mesh, are made into a sufoof (powder). The oil of erandi (Ricinus communis) is then added to the sufoof (Powder). When the proper qiwam (consistency) forms, the medicinal sufoof (powder) is gradually added to it during stirring till both mixes. The Majoon is preserved in glass jar $11,26,27,30$.

\section{Dosage and Administration}

$10 \mathrm{~g}$. with water at bed time $11,20,44,45,46$.

\section{Action and Uses}

Muhallil e Waram (Anti-inflammatory)

Mulayyan (Laxative)

In phlegmatic diseases.

In Waja ul Mafasil (Arthritis), Irq un Nisa (Sciatica), Niqras (Gout) and Qabz (Constipation) 2, 3, 33,44, 45, 46.

\section{Physicochemical Standards of Majoon Suranjan}

Various physicochemical standards of Majoon Suranjan are given below ${ }^{1}$

Table 2: Physicochemical standards of Majoon Suranjan

\begin{tabular}{|l|l|}
\hline Appearance & Semi solid \\
\hline Colour & Moderate Brown \\
\hline Smell & Agreeable \\
\hline Taste & Sweet, Astringent \\
\hline Alcohol soluble matter & $55.92 \%$ \\
\hline Water soluble matter & $62.75 \%$ \\
\hline Successive extractives & \\
Pet. Ether (60-80\%) & $0.50 \%$ \\
Chloroform & $0.33 \%$ \\
Ethyl alcohol & $10.97 \%$ \\
Distilled water & $63.88 \%$ \\
\hline pH of 1\% aq soln & 5.71 \\
\hline pH of 10\% aq soln & 5.71 \\
\hline Bulk density at 25ㅇ & 1.34 \\
\hline Total ash & $1.11 \%$ \\
Water soluble ash & $0.23 \%$ \\
Acid insoluble ash & $0.11 \%$ \\
\hline Volatile oils & $0.28 \% \mathrm{v} / \mathrm{w}$ \\
\hline Alkaloids & $0.27 \%$ \\
\hline Tannins & $0.75 \%$ \\
\hline Resins & $0.58 \%$ \\
\hline Reducing sugars & $52.3 \%$ \\
\hline Crude fibres & $2.6 \%$ \\
\hline Total Nitrogen & $0.42 \%$ \\
\hline Sodium & $18.3 \mathrm{mg} \%$ \\
\hline Potassium & $333.0 \mathrm{mg} \%$ \\
\hline Calcium & $32.3 \mathrm{mg} \%$ \\
\hline Phosphates & $33.7 \mathrm{mg} \%$ \\
\hline & \\
\hline
\end{tabular}

Table 3: Brief Description of Ingredients of Majoon Suranjan

\begin{tabular}{|c|c|c|c|c|c|c|}
\hline Drug & Parts Used & $\begin{array}{l}\text { Botanical } \\
\text { name }\end{array}$ & Dosage & Functions & Uses & Scientific Studies \\
\hline $\begin{array}{l}\text { Barg e Hina } \\
\text { khushk }\end{array}$ & $\begin{array}{l}\text { Leaves }[19,31, \\
50]\end{array}$ & $\begin{array}{l}\text { Lawsonia inermis } \\
{[34,43]}\end{array}$ & $\begin{array}{l}\text { 5gms }[19, \\
31,50]\end{array}$ & $\begin{array}{l}\text { Resolvent, } \\
\text { carminative, } \\
\text { digestive, } \\
\text { emmenogogue } \\
\text { and diuretic }[13 \text {, } \\
21,41,48,50]\end{array}$ & $\begin{array}{l}\text { In colitis, Renal } \\
\text { stones, } \\
\text { Oligouria, and } \\
\text { arthritis }[13,21 \text {, } \\
25,26,30,41,48,50]\end{array}$ & $\begin{array}{l}\text { Antibacterial, antioxidant, } \\
\text { antimicrobial, } \\
\text { hypoglycaemic, } \\
\text { hepatoprotective }[14,23,51]\end{array}$ \\
\hline Badiyan & Seeds $[19,31,50]$ & $\begin{array}{l}\text { Foeniculum } \\
\text { vulgare }[34,43]\end{array}$ & $\begin{array}{l}\text { 6gms [19, } \\
31,50]\end{array}$ & $\begin{array}{l}\text { carminative, } \\
\text { digestive, } \\
\text { galactogogue } \\
\text { and diuretic [13, } \\
21,48,50]\end{array}$ & $\begin{array}{l}\text { In hypertension, } \\
\text { glaucoma, to } \\
\text { improve } \\
\text { eyesight, and in } \\
\text { respiratory and } \\
\text { gastrointestinal } \\
\text { disorders [13,21, } \\
25,26,30,41,48,50]\end{array}$ & $\begin{array}{l}\text { Antibacterial, antioxidant, } \\
\text { anti fungal, } \\
\text { antithrombotic, anti- } \\
\text { inflammatory, } \\
\text { oestorgenic, } \\
\text { hypoglycaemic and } \\
\text { hepatoprotective } \\
\text { activities [15] }\end{array}$ \\
\hline Asarun & $\begin{array}{l}\text { Roots, } \\
\text { Rhizome [19,31, }\end{array}$ & $\begin{array}{l}\text { Valeriana } \\
\text { wallichii }[34,43]\end{array}$ & $\begin{array}{l}\text { 10gms }[19, \\
31,50]\end{array}$ & $\begin{array}{l}\text { Nervine and } \\
\text { brain tonic, }\end{array}$ & $\begin{array}{l}\text { Dementia, } \\
\text { hysteria, }\end{array}$ & $\begin{array}{l}\text { Anti inflammatory, } \\
\text { antispasmodic, }\end{array}$ \\
\hline
\end{tabular}




\begin{tabular}{|c|c|c|c|c|c|c|}
\hline & 50] & & & $\begin{array}{l}\text { Diuretic, } \\
\text { emmenogogue } \\
{[21,48,50]}\end{array}$ & $\begin{array}{l}\text { Paralysis Facial } \\
\text { palsy, Ascites, } \\
\text { Amenorrhoea } \\
{[13,21,25,26,30,48,50]}\end{array}$ & $\begin{array}{l}\text { antimicrobial, analgesic, } \\
\text { psychotic, radioprotective } \\
\text { against radiation injury, } \\
\text { hypnotic, anticonvulsant, } \\
\text { anthelminthic, cytotoxic, } \\
\text { antioxidant. [52] }\end{array}$ \\
\hline $\begin{array}{l}\text { Beekh e } \\
\text { Kibr }\end{array}$ & $\operatorname{Root}[19,31,50]$ & $\begin{array}{l}\text { Caparis spinosa } \\
{[34,43]}\end{array}$ & $\begin{array}{l}5 \text { gms [19, } \\
31,50]\end{array}$ & $\begin{array}{l}\text { Nervine tonic, } \\
\text { Diuretic, anti } \\
\text { inflammatory, } \\
\text { detergent }[21, \\
48,50]\end{array}$ & $\begin{array}{l}\text { Cerebral palsy, } \\
\text { Facial palsy, } \\
\text { Arthritis, Gout, } \\
\text { Ascites, } \\
\text { Lymphadenitis, } \\
\text { Skin diseases } \\
{[13,21,25,26,30,41,} \\
48,50]\end{array}$ & $\begin{array}{l}\text { Anti inflammatory, anti } \\
\text { diabetic, anti obesity, } \\
\text { lowers cholesterol, anti } \\
\text { hypertensive, anti } \\
\text { microbial, antihepatotoxic } \\
\text { [53] }\end{array}$ \\
\hline $\begin{array}{l}\text { Post halela } \\
\text { Zard }\end{array}$ & $\begin{array}{l}\text { Covering of } \\
\text { the fruit }[19,31 \text {, } \\
50]\end{array}$ & $\begin{array}{l}\text { Terminalia } \\
\text { Chebula }[34,43]\end{array}$ & $\begin{array}{l}5 \text { gms [19, } \\
31,50]\end{array}$ & $\begin{array}{l}\text { Brain tonic, } \\
\text { Digestive, gives } \\
\text { strength to } \\
\text { intestines and } \\
\text { stomach }[21,48,50]\end{array}$ & $\begin{array}{l}\text { Joint diseases, } \\
\text { Fevers, Cough, } \\
\text { Asthma, } \\
\text { Dysentery, Colic, } \\
\text { Skin diseases, } \\
\text { Eye diseases } \\
{[13,21,25,26,30,41,} \\
48,50]\end{array}$ & $\begin{array}{l}\text { Anticarcinogenic, } \\
\text { antidiabetic, anti } \\
\text { inflammatory, anti } \\
\text { arthritic, antimicrobial, } \\
\text { antibacterial, antifungal, } \\
\text { antioxidant, } \\
\text { antispasmodic, } \\
\text { hypolipaedimic [12]. }\end{array}$ \\
\hline $\begin{array}{l}\text { Turbud } \\
\text { Safaid }\end{array}$ & $\begin{array}{l}\text { Root and bark } \\
{[19,31,50]}\end{array}$ & $\begin{array}{l}\text { Ipomoea } \\
\text { Turpethum }[34,43]\end{array}$ & $\begin{array}{l}2-4 \text { gms } \\
\text { in } \\
\text { powdered } \\
\text { form }[19,31 \text {, } \\
50]\end{array}$ & $\begin{array}{l}\text { Purgative, } \\
\text { Laxative, } \\
\text { removes } \\
\text { morbid matters } \\
\text { of phlegmatic } \\
\text { and biliary } \\
\text { diseases }[21,48,50]\end{array}$ & $\begin{array}{l}\text { Ascites, Piles, } \\
\text { Arthritis, Gout, } \\
\text { Sciatica, } \\
\text { Melancholia, } \\
\text { Facial palsy, } \\
\text { Hysteria and } \\
\text { Obesity [13,21, 25,26, } \\
30,41,48,50]\end{array}$ & $\begin{array}{l}\text { Anti inflammatory, } \\
\text { Antioxidant, Analgesic, } \\
\text { Anti ulcer, Anti diabetic, } \\
\text { Anti microbial, Anti } \\
\text { diarrhoeal, } \\
\text { hepatoprotective }[8]\end{array}$ \\
\hline $\begin{array}{l}\text { Tukhm } \\
\text { Karafs }\end{array}$ & Seeds $[19,31,50]$ & $\begin{array}{l}\text { Apium graveolens } \\
{[34,43]}\end{array}$ & $\begin{array}{l}\text { 1.2-4 gms } \\
{[19,31,50]}\end{array}$ & $\begin{array}{l}\text { Diuretic, anti } \\
\text { inflammatory }{ }^{[21,} \\
48,50]\end{array}$ & $\begin{array}{l}\text { Ascites, Piles, } \\
\text { Arthritis, Gout, } \\
\text { Sciatica [13,21, 25,26, } \\
30,41,48,50]\end{array}$ & $\begin{array}{l}\text { Antiulcerogenic, } \\
\text { antimicrobial, } \\
\text { anthelminthic, } \\
\text { cardiovascular and } \\
\text { hypolipaedimic, anti } \\
\text { inflammatory [54] }\end{array}$ \\
\hline Chita Lakdi & & $\begin{array}{l}\text { Plumbago } \\
\text { zeylanicum }[34,43]\end{array}$ & $\begin{array}{l}1-1.5 \mathrm{~g} \\
{[19,31,50]}\end{array}$ & $\begin{array}{l}\text { Digestive, } \\
\text { Carminative, } \\
\text { Anti helminthic, } \\
\text { Laxative, } \\
\text { Expectorant } \\
{[13,21,41,48,50]}\end{array}$ & $\begin{array}{l}\text { It is used in } \\
\text { indigestion and } \\
\text { other stomach } \\
\text { ailments. [13,21, } \\
25,26,30,48,50]\end{array}$ & $\begin{array}{l}\text { antimicrobial, anti } \\
\text { inflammatory } \\
\text { antiulcerous, anti diabetic, } \\
\text { Anticancerous, } \\
\text { hypocholestrolemic, } \\
\text { wound healing }[17,38]\end{array}$ \\
\hline Zanjabeel & $\operatorname{Root}[19,31,50]$ & $\begin{array}{l}\text { Zingiber } \\
\text { officinalis }[34,43]\end{array}$ & $\begin{array}{l}1-1.5 \mathrm{~g} \\
{[19,31,50]}\end{array}$ & $\begin{array}{l}\text { Aphrodisiac, } \\
\text { Brain Tonic, } \\
\text { Appetizer, } \\
\text { Digestive, } \\
\text { Carminative, } \\
\text { Anti helminthic, } \\
\text { Laxative, } \\
\text { Expectorant, } \\
\text { Detergent, } \\
\text { Stimulant. }[13,21 \text {, } \\
41,48,50]\end{array}$ & $\begin{array}{l}\text { It is used in } \\
\text { sexual weakness, } \\
\text { paralysis, Bell's } \\
\text { palsy. It is } \\
\text { also used in } \\
\text { indigestion and } \\
\text { other stomach } \\
\text { ailments. [13,21, } \\
25,26,30,48,50]\end{array}$ & $\begin{array}{l}\text { Cardio tonic, Powerful } \\
\text { Antioxidant, Anti-cancer, } \\
\text { Anti coagulant, Anti } \\
\text { inflammatory, Anti } \\
\text { atherosclerotic, Antacid, } \\
\text { Antimicrobial, Antitussive } \\
\text { and Immuno -modulatory. } \\
\text { [47] }\end{array}$ \\
\hline Saqmunia & $\operatorname{Gum}[19,31,50]$ & $\begin{array}{l}\text { Convolvulus } \\
\text { scammonia }[34,43]\end{array}$ & $\begin{array}{l}1-1.5 g[19 \\
31,50]\end{array}$ & $\begin{array}{l}\text { Purgative, anti } \\
\text { inflammatory, } \\
\text { anthelminthic } \\
{[21,41,48,50]}\end{array}$ & $\begin{array}{l}\text { Severe } \\
\text { constipation, } \\
\text { Ascites, } \\
\text { Osteoarthritis } \\
{[13,21,25,26,30,48,50]}\end{array}$ & $\begin{array}{l}\text { Cytotoxic effect, } \\
\text { Vasorelaxation and anti } \\
\text { platelet aggregation, } \\
\text { Anticancerous [16] }\end{array}$ \\
\hline $\begin{array}{l}\text { Samundar } \\
\text { Jhag }\end{array}$ & & Cuttlefish bone & $\begin{array}{l}1-2 \text { gms } \\
{[19,31,50]}\end{array}$ & $\begin{array}{l}\text { Detergent [21, } \\
48,50]\end{array}$ & $\begin{array}{l}\text { Eye diseases, } \\
\text { Skin diseases } \\
{[13,21,25,26,30,48,50]}\end{array}$ & $\begin{array}{l}\text { Anti microbial, Anti } \\
\text { oxidant[37] }\end{array}$ \\
\hline $\begin{array}{l}\text { Suranjan } \\
\text { Shireen }\end{array}$ & $\operatorname{Root}[19,31,50]$ & $\begin{array}{l}\text { Colchicum luteum } \\
{[34,43]}\end{array}$ & $\begin{array}{l}4 \text { gms [19, } \\
31,50]\end{array}$ & $\begin{array}{l}\text { Purgative, anti } \\
\text { inflammatory, } \\
\text { analgesic, } \\
\text { diuretic [21, } 41 \text {, } \\
48,50]\end{array}$ & $\begin{array}{l}\text { Arthritis, } \\
\text { Sciatica, Gout [2, } \\
13,21,25,26,30,48,50]\end{array}$ & $\begin{array}{l}\text { Anti inflammatory, Anti } \\
\text { arthritic }[6,24]\end{array}$ \\
\hline Sana Makki & $\begin{array}{l}\text { Leaves }[19,31 \text {, } \\
\text { 50] }\end{array}$ & $\begin{array}{l}\text { Cassia } \\
\text { angustifolia }[34,43]\end{array}$ & $\begin{array}{l}\text { 7-10 gms } \\
{[19,31,50]}\end{array}$ & $\begin{array}{l}\text { Purgative [21, } \\
48,50]\end{array}$ & $\begin{array}{l}\text { Severe } \\
\text { constipation } \\
{[13,21,25,26,30,48,50]}\end{array}$ & $\begin{array}{l}\text { Anti microbial, Anti } \\
\text { oxidant:[25] }\end{array}$ \\
\hline Satar Farsi & & $\begin{array}{l}\text { Origanum vulgare } \\
{[34,43]}\end{array}$ & $\begin{array}{l}5 \text { gms }[19, \\
31,50]\end{array}$ & $\begin{array}{l}\text { Digestive, } \\
\text { Carminative, } \\
\text { Expectorant [13, }\end{array}$ & $\begin{array}{l}\text { Gastritis, } \\
\text { Flatulence, } \\
\text { Hiccup, }\end{array}$ & $\begin{array}{l}\text { Anti oxidant, anti } \\
\text { inflammatory, anti cancer, } \\
\text { anti bacterial [37] }\end{array}$ \\
\hline
\end{tabular}




\begin{tabular}{|c|c|c|c|c|c|c|}
\hline & & & & $21,48,50]$ & $\begin{array}{l}\text { Bronchial } \\
\text { Asthma }[13,21,25,26 \text {, } \\
30,48,50]\end{array}$ & \\
\hline Filfil Safaid & Fruit $[19,31,50]$ & $\begin{array}{l}\text { Piper album linn } \\
{[34,43]}\end{array}$ & $\begin{array}{l}\text { 1-2 gms } \\
{[19,31,50]}\end{array}$ & $\begin{array}{l}\text { Carminative, } \\
\text { Anti fertility, } \\
\text { Diuretic } \\
\text { as well as } \\
\text { emmenagogue, } \\
\text { Haeminic, } \\
\text { Aphrodisiac, } \\
\text { Stomachic, } \\
\text { Analgesic. } \\
{[13,21,48,50]}\end{array}$ & $\begin{array}{l}\text { It is used in cold } \\
\text { and other } \\
\text { respiratory } \\
\text { disorders, } \\
\text { paralysis, Bell's } \\
\text { palsy, arthritis, } \\
\text { cough, sciatica, } \\
\text { gout, night } \\
\text { blindness, } \\
\text { epilepsy, and } \\
\text { asthma [13,21, 25,26, } \\
\text { 30,48,50] }\end{array}$ & $\begin{array}{l}\text { Scientific reports are } \\
\text { available for Anti stress, } \\
\text { Antitumor, Anti diabetic, } \\
\text { Antimicrobial, Anti fungal } \\
\text { activity, Anti Tubercular } \\
\text { activity, } \\
\text { Anti inflammatory, Anti } \\
\text { arthritic, Antioxidant, } \\
\text { Antifertility, Hepato- } \\
\text { protective and Cardio- } \\
\text { protective activity.[25] }\end{array}$ \\
\hline $\begin{array}{l}\text { Kishneez } \\
\text { Khushk }\end{array}$ & $\begin{array}{l}\text { Whole plant } \\
\text { and fruit }[19,31 \text {, } \\
50]\end{array}$ & $\begin{array}{l}\text { Coriandrum } \\
\text { sativum }[34,43]\end{array}$ & $\begin{array}{l}10 \text { gms } \\
{[19,31,50]}\end{array}$ & $\begin{array}{l}\text { Analgesic, Anti } \\
\text { inflammatory } \\
{[13,21,41,48,50]}\end{array}$ & $\begin{array}{l}\text { Stomatitis, } \\
\text { Burning } \\
\text { micturition, } \\
\text { quenches thirst, } \\
\text { Tonsillitis, } \\
\text { Lymphadenitis, } \\
\text { vertigo and } \\
\text { giddiness [13,21, } \\
25,26,30,48,50]\end{array}$ & $\begin{array}{l}\text { Antioxidant, Anti diabetic, } \\
\text { Hepato- protective, } \\
\text { Antimicrobial, Anti fungal } \\
\text { activity, Anticancerous, } \\
\text { anthelminthic, anti } \\
\text { convulsant, anti } \\
\text { mutagenic, diuretic }{ }^{[40]}\end{array}$ \\
\hline Gul e Surkh & $\begin{array}{l}\text { Petals and } \\
\text { rhizomes }[19, \\
31,50]\end{array}$ & $\begin{array}{l}\text { Rosa damascena } \\
{[34,43]}\end{array}$ & $\begin{array}{l}10-20 \\
\text { gms [19, 31, } \\
50]\end{array}$ & $\begin{array}{l}\text { Anti } \\
\text { inflammatory, } \\
\text { laxative, } \\
\text { haemostatic [13, } \\
21,48,50]\end{array}$ & $\begin{array}{l}\text { Hepatitis, } \\
\text { haemoptisis, } \\
\text { Constipation, } \\
\text { Conjunctivitis, } \\
\text { Palpitation [13,21, } \\
25,26,30,48,50]\end{array}$ & $\begin{array}{l}\text { Analgesic, hypnotic, anti } \\
\text { convulsant, Anti diabetic, } \\
\text { Antimicrobial, } \\
\text { Antioxidant, Anti } \\
\text { inflammatory, laxative, } \\
\text { anti ageing [39] }\end{array}$ \\
\hline
\end{tabular}

Various functions of Majoon Suranjan is attributed to its various ingredients which possess antioxidant, hematogenic, body building, stomachic, anti inflammatory, aphrodisiac, adipogenous, carminative, tonic for nerves, brain, heart and kidneys, nerve stimulant, cicatrizant, emmenagogue, laxative, expectorant, demulcent, antipyretic, blood purifier, antitussive etc properties.

\section{REFERENCES}

1. Anonymous. "Physicochemical standards of Unani formulations". Part 1st. New Delhi, CCRUM, (1986). pp - 90.

2. Anonymous. "National Formulary of Unani Medicine" Vol. V. New Delhi: Ministry of H \& FW (2008). Pp - 106.

3. Anonymous. "The Unani Pharmacopoiea of India". Part 1, Vol I, New Delhi, AYUSH, CCRUM: pp - 56, 57, 76, 77, 88, 89.

4. Anonymous. "Qarabadeen Sarkari" Ministry of Health \& Family welfare, (YNM). Pp - 181-182.

5. Anonymous. "Qarabadeen Azam wa Akmal", published by CCRUM, New Delhi (YNM). Pp - 472.

6. Abed, K. F. Antimicrobial activity of essential oils of some medicinal plants from Saudi Arabia. Saudi J. Biol. Sci. 2007; 14:53-60.

7. Ali G.A. "Ilaj ul Gurba" Idarah Kitab us shifa, New Delhi, pp 153-157

8. Ahmad T, Tariq M, Siddiqui J. I, Ahmed M.W., Kazmi M. H. "A Review on Operculina turpethum: A Potent Herb of Unani System of Medicine". Journal of Pharmacognosy and Phytochemistry 2017; 6(1):23-26.

9. Arzani H. M. A, "Qarabadeen Quadri": Munshi Nawal Kishore, Kanpur, (1268). pp 453.

10. Arzani H. M. A. "Tibb e Akbar" Idarah Kitab Us shifa, New Delhi, pp - 614-617.

11. Arzani H. M. A. "Meezan ul Tib" (Urdu), 1 $1^{\text {st }}$ Edition: pp-178-81, 756.

12. Ashwini, R. Gajalakshmi S., Mythili S., Sathiavelu A. "Terminalia Chebula- A pharmacological review" R. Ashwini et al. / Journal of Pharmacy Research 2011; 4(9):2884-2887

13. Baitar I. "Kitab-ul-Jamia Al Mufridaat al Advia wal Aghzia". 1 $1^{\text {st }}$ Edition, New Delhi. CCRUM, (1985); pp - 130,277.

\section{CONCLUSION}

With the above discussion the inference may be drawn that the Majoon Suranjan is one of the best Unani formulation with a lot of health benefits. It has proven to be beneficial in treatment of Arthritis. However more scientific studies and clinical trials are needed on this compound formulation to ensure its scientific validation for clinical use in patients in general.
14. Chaudhary G.D, Poonia P, Kamboj P and Kalia A.N. Hepatoprotective potential of Lawsonia inermis (seeds). Int J Phytopharmacology(2012; 3:66-73

15. Choi, E.M., Hwang, J. K. "Anti-inflammatory, analgesic and antioxidant activities of the fruit of Foeniculum vulgare". Fitoterapia 2004; 75:557-565.

16. Esmail A., Snafi A. "The chemical constituents and pharmacological effects of Convolvulus arvensis and Convolvulus scammonia- A review" IOSR Journal of Pharmacy Volume 6, Issue 6 Version. 2016; 3: 64-75

17. Ganesan K, Gani, S. B. "Ethnomedical and Pharmacological Potentials of Plumbago zeylanica L- A Review". American Journal of Phytomedicine and Clinical Therapeutics.

18. Ghani N. H, "Qarabadeen Najmul Ghani". New Delhi CCRUM, (YNM), pp - 692

19. Ghani N. H. "Khazainul Advia". New Delhi: Idara Kitabul Shifa; (YNM), 187-188, 421-422, 869, 682, 1284, 1231-31, 495, 756$57,1097,605-6,542-43$.

20. Hassan B.A., Ali bin H. "Kitab al Mukhtaraat fil Tibb". Part 2nd. CCRUM New Delhi: (2005), pp -310.

21. Hakim M A. "Bustanul Mufredat". New Delhi: Idara Kitabus Shifa; (2002) pp - 90, 145, 60, 266, 558, 180, 316, 249, 470, 235-36, 209.

22. Hameed H. A, Said H. M. "Hamdard Pharmacopoeia of Eastern medicine". Delhi: Sri Satguru publications (1997), pp - 274275.

23. Habbal. O, Hassan S. S. "Antibacterial activity of Lawsonia inermis Linn (Henna) against Pseudomonas aeruginosa". Asian Pacific journal of Tropical Biomedicine. 2011; 1(3). 
24. Javed M, Khan J. A, Siddiqui M. M. H., "Effects of colchicum luteum baker in the management of Arthritis". Indian Journal of Traditional Knowledge. 2005; 4(4):421-423.

25. Jurjani A. H, "Zakhira Khawarzam Shahi”, (Urdu), 1 $1^{\text {st }}$ Edition, New Delhi, Idarah Kitab Us shifa: pp - 635-36.

26. Kabeeruddin H. M, "Al Qarabadeen". CCRUM New Delhi, (2006). Pp-1212.

27. Kabeeruddin H. M, “Al Qarabadeen". Vol II. Malik son's publishers, Faisalabad, (YNM). Pp-413.

28. Kabeeruddin H. M, "Bayaz e Kabeer". Vol. 1. Hyderabad: Hikmat Book Depot, (YNM) pp-137.

29. Kabeeruddin H. M, "Ikseer e Azam", (Urdu translation), Vol 2, Ejaz Publishing House, New Delhi, pp -1430-1447.

30. Kabeeruddin H. M, "Sharah Asbab” Vol III, Shaukat Book Depot, Gujrat, (YNM). Pp-180-186.

31. Kabeeruddin M. "Makhzen ul Mufredat". New Delhi: Ejaz Publishing House; (YNM), pp-108, 145, 146, 366, 279, 560, 538, 178, 311-13, 251-52, 574, 239, 212.

32. Khan M. A, "Qarabadeen-e-Azam", Aijaz Publishing House, New Delhi. (1957), pp- 576.

33. Khan M. A. "Moheet-e-Azam". Vol. 1. New Delhi: CCRUM. (2012); pp - 213-215.

34. Khare C.P. "Indian Medicinal Plants: an illustrated dictionary". New Delhi: Springer (India) Private Limited; (2007) pp - 238, $400,652,653,735,150-151,711-12,492,491,501,363-64$, $163-64,488,452$.

35. Lutfi S. A, "Qarabadeen Lutfi". Delhi: Mutbua Ghulam Nizamuddin (1924), pp 3-342.

36. Majusi I. A, "Kamil us Sana”, (Urdu), Vol 2. New Delhi, Idarah Kitab Ushifa: pp-277.

37. Masoudi M, Saiedi M. "Anti-inflammatory, Antioxidant, anticancer and anti-microbial effect of Origanum vulgare: a systematic review". Scholars Research Library Der Pharmacia Lettre, 2017; 9 (4):85-94

38. Mandavkar Y.D, Jalalpure S.S. "A comprehensive review on Plumbago zeylanica Linn". African Journal of pharmacy and pharmacology 2011; 5(25):2738-2747.

39. Mahboubi M. "Rosa damascena as holy ancient herb with novel applications. Journal of Traditional Complementary Medicine". 2016; 6(1);
40. Momin A H., Acharya S. S, Gajjar A. V. "Coriandrum Sativum Review of Advances in Phytopharmacology". International Journal Of Pharmaceutical Sciences And Research

41. Multani H.C. "Hindustan aur Pakistan ki Jadi Bootiyan aur unke Fawaid”. Lahore: Maktaba Daniyal. (YNM) pp - 19, 22, 561-63, 1006, 1009, 1012, 1009.

42. Nadwi R. A., "Firdaus ul Hikmat”, Urdu, New Delhi, CCRUM: pp634 .

43. Nadkarni A. K. "Indian Materia Medica with Ayurvedic, UnaniTibbi, Siddha, Allopathic, Homeopathic, Naturopathic and Home remedies". Vol. 1st. Bombay: Popular Prakashan Private Ltd; (2009) pp - 480, 772, 1203, 1309, 333, 1285-86, 969-72, 965, 140, 991-92, 957, 873-74.

44. Qamari A. "Geena Muna" published by CCRUM, New Delhi, (2007). pp - 339, 341

45. Raazi Z, "Kitab al Hawi", (Urdu), vol 11, New Delhi, CCRUM, (2004): pp -75-85, 127.

46. Raazi Z, "Kitab ul Mansoori”, New Delhi, CCRUM, pp - 391-393.

47. Rehman $\mathrm{R}$, Akram. M. "Zingiber officinale Roscoe (pharmacological activity)" Journal of Medicinal Plants Research, 5(3):344-348.

48. Said H. M, "Al Biruni's book on Pharmacy and Materia Medica". Karachi: Hamdard National Foundation, (1973), pp - 38-50.

49. Sina I, "Alqanoon Fil Tibb", (Urdu), Vol III (Part 2), Aijaz Publishing House, New Delhi: pp 1119 - 1120.

50. Safiuddin A, "Unani Advia Mufreda", Qaumi Council Barae farogh Urdu Zaban. (2004) 92: pp-153, 175.

51. Syamsudin I, Winarno H. "The effects of Inai (Lawsonia inermis) leave extract on blood sugar level: An Experimental Study". Res J Pharmacol, 2008; 2(2):20-22

52. Shetty S, Bhatt N. "A comparative clinical study on the effect of Tagara (Valeriana wallichii DC.) and Jatamansi (Nardostachys jatamansi DC.) in the management of Anidra (primary insomnia). Pp- 212- 217.

53. Zhang H. "Phytochemical and Pharmacological Properties of Capparis spinosa as a Medicinal Plant”. Nutrients: (2018); 10 (2).

54. Znafi A. E. "The Pharmacology of Apium graveolens. -A Review". International Journal for Pharmaceutical Research Scholars 2014; 3(1): 671-677. 